\title{
Statistically Filtering Data for Operational Modal Analysis under Ambient Vibration in Structural Health Monitoring Systems
}

\author{
Liang Wang, Ruixiang Song, Yubin Wu, Wencheng $\mathrm{Hu}$ \\ Beijing Municipal Institute of Labour Protection, 55 Taoranting Road, Xicheng District, Beijing 100054, China
}

\begin{abstract}
Operational modal analysis (OMA) is prevalent in large structure modal identification for that it asks for output measurements only. To guarantee identification accuracy, theoretically, OMA data need to be a random process of Gaussian white noise $(\mathrm{GWN})$. Although numerous OMA applications are found in practice, few have particularly discussed the data distribution and to what extent it would blur the modal judgement. This paper presents a method to sieve segments mostly obeying the GWN distribution out of a recording. With a windowing technique, the data segments are evaluated by the modified Kurtosis value. The process has been demonstrated on the monitoring data of two case study structures: one is a laboratory truss bridge excited by artificial forces, the other is a real cable-stayed bridge subject to environmental loads. The results show that weak randomness data may result in false peaks that would possibly mislead the non-parametric modal identification, such as using the Frequency Domain Decomposition method. To overcome, cares on selecting the optimal segment shall be exercised. The proposed method is verified effective to find the most suitable data for modal identification of structural health monitoring systems.
\end{abstract}

\section{Introduction}

Operational modal analysis (OMA) has been extensively applied in modal tests of practical structures due to its convenient output-only merit. In bridge monitoring projects, for example, a well-known example is the WASHMS system which has mounted more than 800 sensors to monitor and identify the operational condition of three landmark bridges in Hong Kong [1]. Also, Shama et al. [2] conducted ambient vibration tests on North Grand Island Bridge in West New York for the FE model validation. Brown john et al. [3] acquired the OMA data of the Humber Bridge in 2008 and studied the modal parameter variation from an old dataset obtained decades ago. The OMA techniques are also safe and suitable for the modal test of historical architecture. Relevant applications include an ancient Cypriot aqueduct built in 1747 [4], a Tunisian temple built in16th century [5], [6], and an Egyptian Al-Sultaniyamirarets built in 1337 [7].Moreover, the rapid increase of the research in theory and applications of this area has motivated the creation of the International Operational Modal Analysis Conference (IOMAC) since 2005.

During an OMA test session the input is natural excitation such as traffic, wind, and other natural loads, and all OMA methods attempt to extract low noise information from the measured output. Popular approaches include frequency domain decomposition (FDD), covariance/data driven stochastic subspace identification (SSI-COV/-DATA), poly-least squares complex frequency domain (p-LSCF), maximum likelihood (ML) identification, and instrumental variable (IV) method etc. [8].

No matter whether a method is in time or frequency domain, one assumption of the OMA technique must be fulfilled: the responses of the underlying structure excited by natural loads are Gaussian White Noise (GWN). In literature, however, many have simply assumed that the data of adequately long time recording is supposed to be a white noise. Few have further investigated the measured data's randomness and its influence to modal identification results. In order to check the distribution characteristics of the output measurement and help analysts select the data segments that most likely have reliable identification results. To meet this need, the paper proposes a statistical index of the modified kurtosis to judge data randomness. The data selection process based on the index is illustrated by the ambient vibration data acquired a laboratory truss bridge (Case-study I) and a practical cable-stayed bridge (Case-study II). In addition, a windowing judgement strategy is adopted to locate the optimal data segment for subsequent analysis. In the study FDD method has been employed to determine the resonance of the structure. The comparative results showed that the data with weak randomness may affect the modal identification judgment, and the proposed method is straightforward to implement. Optimal data segments are filtered out in both case studies. 


\section{Frequency domain decomposition}

FDD is a non-parametric frequency domain method extended from the basic frequency domain (BFD) technique[9] where resonance is subjectively determined by identifying spikes in singular value decomposition spectra. Consider the response $y(t)$ of a time-invariant, light damped structure being decomposed into different modes $\Phi$ in modal coordinates $q(t)[10]$

$$
y(t)=\Phi q(t)
$$

The correlation between the response $y(t)$ of the current and $\tau$ steps afterwards is given by Eq.(2)

$$
\begin{aligned}
& C_{y y}(\tau)=E\left[y(t+\tau) y(t)^{T}\right] \\
& =E\left[\Phi q(t+\tau) q(t)^{H} \Phi^{H}\right]=\Phi C_{q q}(\tau) \Phi^{H}
\end{aligned}
$$

where $(\bullet)^{H}$ denotes the conjugate transposition of a vector or matrix and $\mathrm{E}[\bullet]$ the mathematical expectation. Fourier transformation of Eq.(2) gives

$$
G_{y y}(\omega)=\Phi G_{q q}(\omega) \Phi^{H}
$$

where $\mathrm{G}_{q q}(\omega)$ is the spectrum matrix of the modal coordinates. $\mathrm{G}_{y y}(\omega)$ is the spectrum matrix of the response that can be estimated from the auto-power spectral density and the cross-power spectral density by the Welch's method [11]. Singular value decomposition (SVD) of the measured response spectrum is written as

$$
\hat{G}_{y y}(\omega)=V S V^{H}
$$

It is noticed that Eq.(4) has a similar form as Eq.(3). Therefore, the diagonal matrix $\mathrm{S}$ is an estimate for modal coordinates $\mathrm{G}_{q q}(\omega)$ while the corresponding column in $V$ is an estimate for $\Phi$. If the input is a GWN in time and space, the estimated modal coordinates are uncorrelated, and the columns are orthogonal to each other. Under this context, once the SVD is calculated, mode shapes are estimated at the frequencies of singular vector peaks, and the frequencies associated are natural frequencies. This is because the modes at these peaks are most likely independent and the modal estimation is reliable. However, it is worth noted that such modal estimation would be invalid if the input fails to follow a GWN distribution. Modal identification using the FDD method is illustrated in Fig. 5 and Fig. 8 as examples.

\section{Kurtosis-based data selection}

Having noticed the importance of the "quality" of an acceleration time-series in terms of statistical distribution, checking the Kurtosis value of the recording is helpful to select the most optimal segment out of standalone measurement. Statistically, Kurtosis measures the peakedness of the probability density function (PDF) of a real-valued random variable, and is the fourth moment of the data or population scaled to the fourth power of its standard deviation [12].

$$
\gamma(x \mid \mu, \sigma)=\frac{E\left[(x-\mu)^{4}\right]}{\sigma^{4}}
$$

where $\mu$ and $\sigma$ is the mean and the standard deviation of series $x$, respectively. In theory, Kurtosis of a normal distribution is equal to three. In this paper, instead, a modified Kurtosis described in Eq. (6) is used.

$$
\gamma^{*}(x \mid \mu, \sigma)=\frac{E\left[(x-\mu)^{4}\right]}{\sigma^{4}}-3
$$

If a dataset is of GWN, the modified Kurtosis should be zero and it is ideal for modal identification. However, since data acquired from the real-world are always subject to environmental unknown errors, even the data recorded for a long period may not render a zero Kurtosis. Practically the analyst has to select the segment with the minimal Kurtosis to represent the structure's current condition. For this reason, the research adopts a windowing strategy to select the most appropriate data segment in terms of its Kurtosis value.

To begin, detrend the raw time-series data and divide it into overlapping segments in adequate length. A minimum segment length, for instance, suggested by SVS [13] is $T_{\min }=1000 / f_{\min }$ where $f_{\min }$ is the lowest natural frequency of interest of the underlying structure. In the whole time-series, the segment $\operatorname{seg}_{i}$ overlaps with its neighbouring counterparts by $w$ per cent. An overlapping factor is set to control the interval of moving "segment windows". The total number of segments equals to

$$
n \operatorname{seg}=\frac{L-\operatorname{seg} L}{(1-w) \operatorname{seg} L}
$$

where $L$ is the length of raw time-series and $\operatorname{seg} L$ the length of each segment.

Next, calculate the modified Kurtosis for each segment and plot a Kurtosis time-history graph for all the segments. Since most often a test session is likely to have more than one channel, we use an averaged Kurtosis index to check the randomness. Then the optimal data segment is selected and used for subsequent processes.

\section{Case-study I: Laboratory truss bridge}

A laboratory steel truss bridge shown in Fig. 1 is a scaled model established to study structural health monitoring systems [14]. OMA tests were conducted and the FDD method was adopted to obtain modal properties. Ambient natural loads were artificially simulated by powerful floor fans blowing in random directions. Due to the limited number of sensors, eight sensor layouts were set to reach all the locations as depicted in Fig. 5 (LVT3 8 as example). For some layouts two sessions were recorded, such as LVT1-1, LVT1-2, ... LVT6-1, LVT6-2.Each session consists of 9 channels. Among the layouts two channels $(\mathrm{CH} 1$ and $\mathrm{CH} 2)$ were fixed while the other is roving. Upon the completion of the test, all layouts were combined to profile the mode shapes of the structure. 


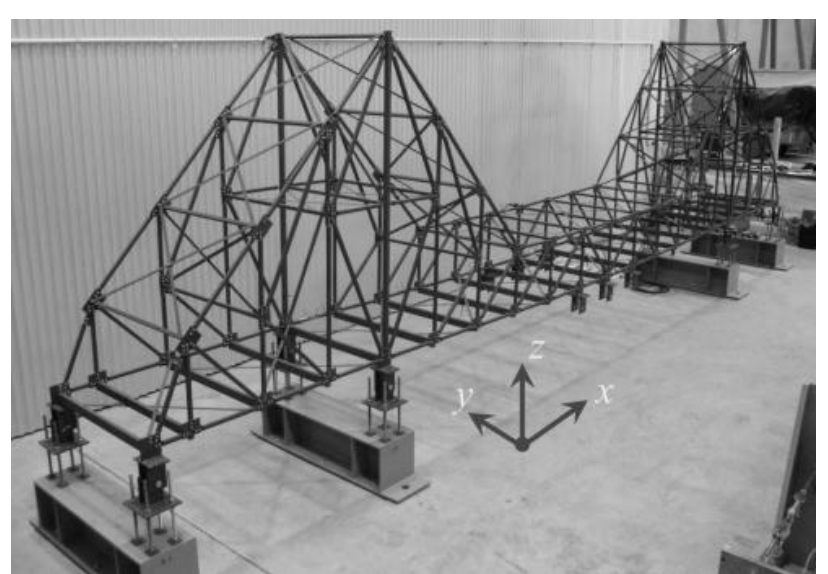

Figure 1. Configuration of the laboratory truss bridge model.

In practice, randomness assumption is often valid if a time-series has a small Kurtosis and the data is suitable for post-processing. Fig. 2 shows the result using Kurtosis to check the raw data of the model. It is found that Kurtosis for most layouts is less than 1.0 except for LVT8 which has a relatively high value. Hence,further data selection for this layout is required.

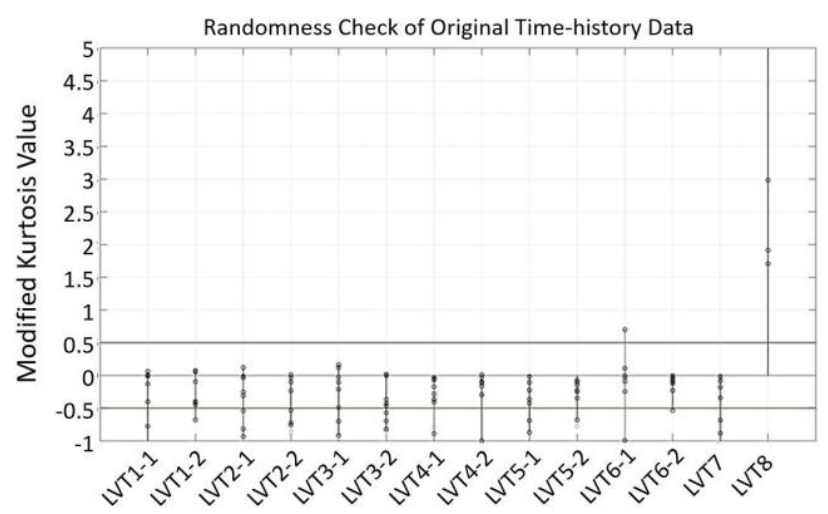

Figure 2. Kurtosis check of acceleration time-history.

\subsection{Data selection and the windowing strategy}

To apply the OMA technique, adequate length of data of the complete 450 seconds is necessary. In this case, preanalysis indicated that the lowest natural frequency is 6.5 $\mathrm{Hz}$, which leads to a minimum segment length ( $\mathrm{seg} L$ ) of $1000 / 6.5=154$ seconds. The sampling frequency is $883.4 \mathrm{~Hz}$ which is adequate for the interested frequency up to $200 \mathrm{~Hz}$ [15]. The overlapping ratio $w$ was $95 \%$. Take layout LVT3-2 as an example, Fig. 3 highlighted the segment with the minimum Kurtosis value equal to 0.3 . Here the plotted Kurtosis was the average of all channels and the blue dotted line located the beginning point of the optimal segment. In the bottom time history, outliers are found in the beginning where the segments' Kurtosis was very high, which means that impacts during a test have substantially affected the randomness.

In addition, Table 1 lists the modified Kurtosis of the complete time-series against the optimal segment for all roving sensor layouts. Among them, for LVT 2-2, 3-2, and LVT8 Kurtosis of the best segment is $20 \%$ less than that of the whole data session.

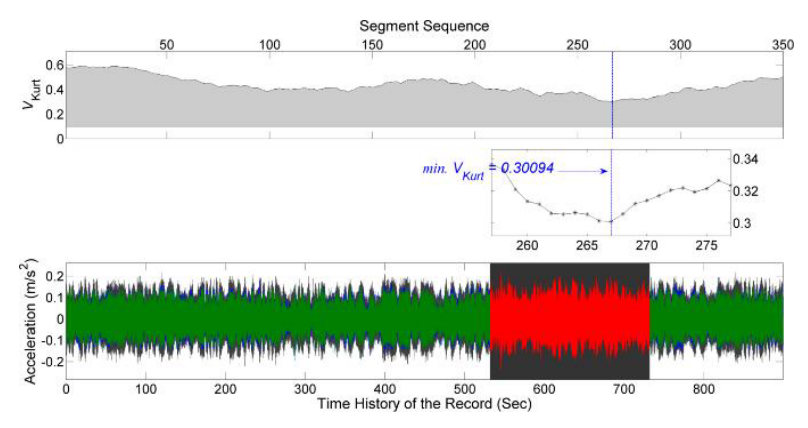

Figure 3. Time-history of data's Kurtosis of layout LVT8.

Table 1. Modified Kurtosis of whole and optimal data segment.

\begin{tabular}{cccc}
\hline \multirow{2}{*}{ Test Session } & \multicolumn{3}{c}{ Modified Kurtosis } \\
\cline { 2 - 4 } & Whole & Optimal & Error \\
\hline LVT1-1 & 0.448 & 0.463 & $3.2 \%$ \\
LVT1-2 & 0.342 & 0.301 & $12.0 \%$ \\
LVT2-1 & 0.471 & 0.459 & $2.5 \%$ \\
LVT2-2 & 0.386 & 0.295 & $23.6 \%$ \\
LVT3-1 & 0.411 & 0.390 & $5.1 \%$ \\
LVT3-2 & 0.468 & 0.301 & $35.7 \%$ \\
LVT4-1 & 0.339 & 0.329 & $2.9 \%$ \\
LVT4-2 & 0.236 & 0.220 & $6.8 \%$ \\
LVT5-1 & 0.382 & 0.369 & $3.4 \%$ \\
LVT5-2 & 0.300 & 0.277 & $7.7 \%$ \\
LVT6-1 & 0.380 & 0.324 & $14.7 \%$ \\
LVT6-2 & 0.148 & 0.123 & $16.9 \%$ \\
LVT7 & 0.494 & 0.480 & $2.8 \%$ \\
LVT8 & 8.156 & 0.050 & $99.4 \%$ \\
\hline
\end{tabular}

In order to further reveal the influence of the data randomness, the SVD spectra used in FDD technique made by the data segment of the lowest and highest Kurtosis of layout LVT8 were compared in Fig. 4.

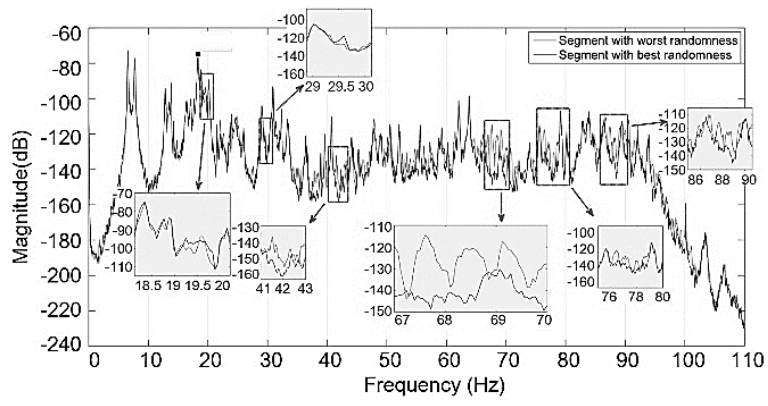

Figure 4. SVD spectra made by best/worst data segments in terms of randomness.

In Fig. 4, obvious discrepancy has been found under $20 \mathrm{~Hz}$, and unmatched peaks of the segments with the highest and the lowest Kurtosis existed at many frequency points. Some peaks disappeared or shifted in the spectrum. The comparison indicates that careless selection of the data segment, especially where the unwanted impacts are involved, may lead to inaccurate resonance judgment in the non-parametric OMA methods.

\section{Case-study II: Cable-stayed bridge}

Another case-study was the application of the selection method on a 25 -year cable-stayed bridge in north China. 
This SHM-instrumented cable-stayed bridge is regarded as a benchmark structure as shown in Fig. 6 [16]. 16channel of acceleration are monitored at the sampling frequency of $100 \mathrm{~Hz}$. In the study, a 24-hour dataset of "Damage status 1" were selected to execute the randomness check.
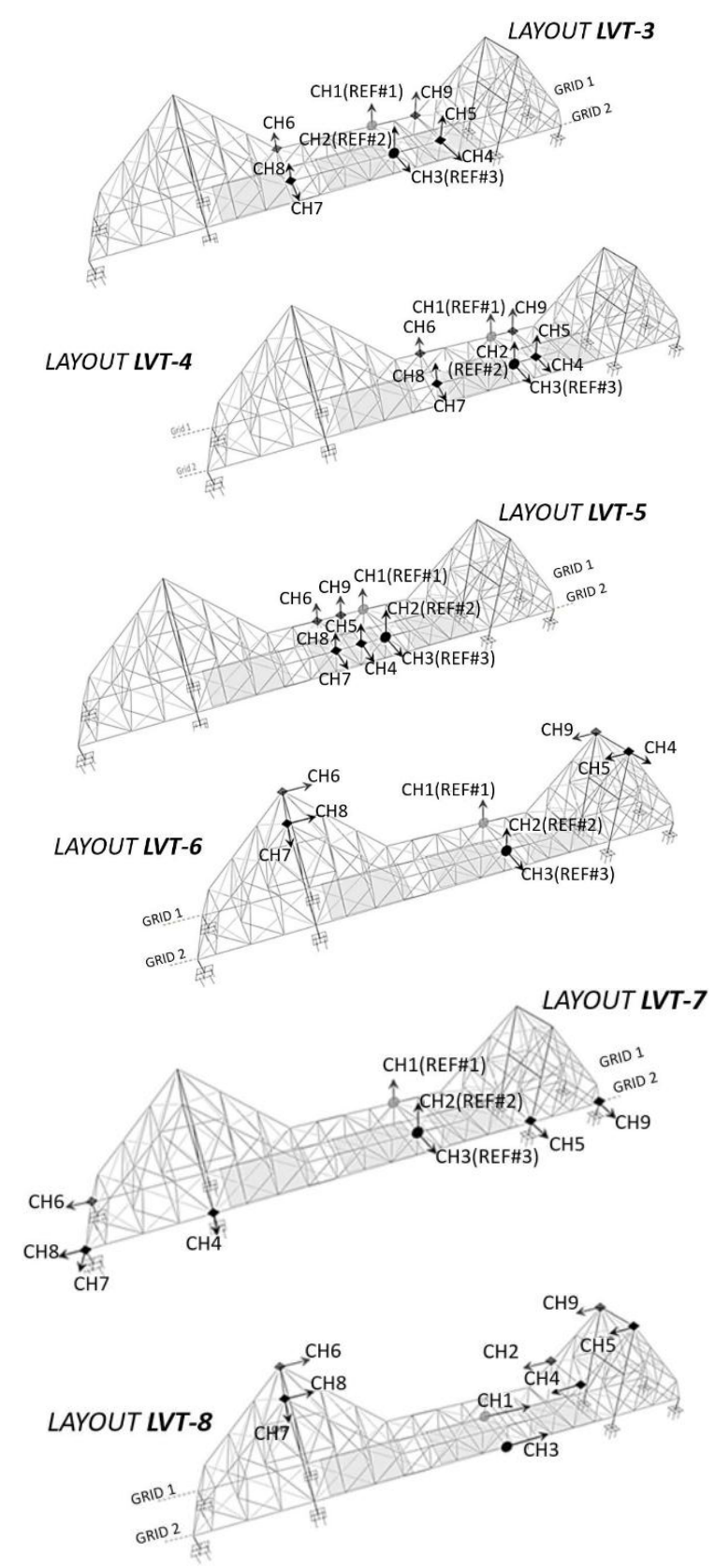

Figure 5. Eight sensor layouts (LVT3-LVT8) used in the ambient vibration tests in Case-study I.

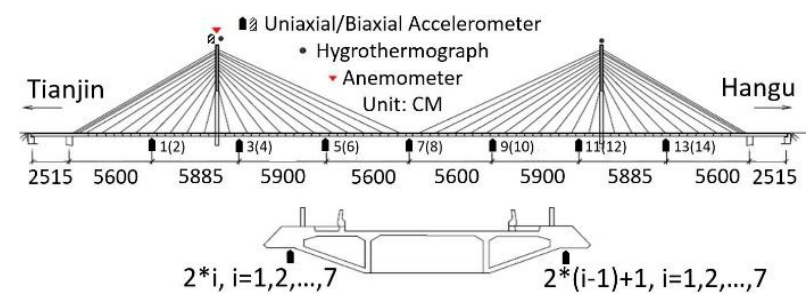

Figure 6. Sensor layout of the case-study cable-stayed bridge [15].
The analytical lowest natural frequency of the structure is $0.201 \mathrm{~Hz}$ [15], which means the minimum segment length is 1.39 hours. Applying the windowing strategy, Fig. 7 indicates the result of the optimal data segment. Since the highest frequency of interest for the flexible structure is $2.5 \mathrm{~Hz}, 20 \mathrm{~Hz}$ was chosen as the sampling frequency [15].

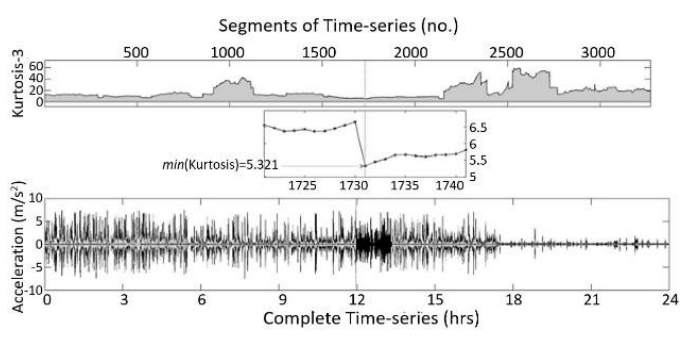

Figure 7.Selected data segment of lowest Kurtosis for "Damage status 1" data.

Applying the windowing strategy, the lowest modified Kurtosis is 5.3 while the maximum is around 60 . Interestingly, we found the data from the mid-night have higher Kurtosis values than the segments of other times. This is contrary to the pre-knowledge that the structure in the night-time would more likely to have natural excitation. Besides, the bottom graph in Fig. 7 shows small impacts probably perhaps caused by off-peak heavy vehicles. Even though the magnitudes are small comparing to that in the daytime, these impacts may render low-Kurtosis data and are hence inappropriate for post-processing.SVD results from the worst, best, and complete 24-hour data have been compared together in Fig. 8 .

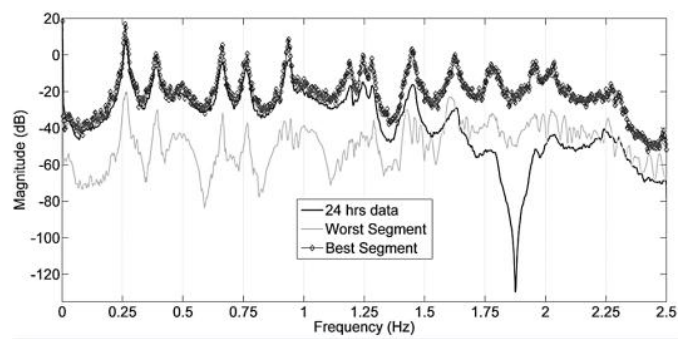

Figure 8. Spectrum of SVD values in FDD using different data segment of the case-study II structure (Data: Damage status 1)

Table 2. Identified natural frequencies by peak picking the spectrum in Figure $8(\mathrm{~Hz})$.

\begin{tabular}{ccccc}
\hline \multirow{2}{*}{ Mode } & \multicolumn{2}{c}{ Identified natural frequencies $(\mathrm{Hz})$} & \multirow{2}{*}{ Error } \\
\cline { 2 - 4 } & Worst & Whole & Best & \\
\hline 1 & 0.2661 & 0.2637 & 0.2612 & $1.84 \%$ \\
2 & 0.3931 & 0.3906 & 0.3882 & $1.25 \%$ \\
3 & 0.6641 & 0.6641 & 0.6641 & 0 \\
4 & 0.7666 & 0.7593 & 0.7666 & 0 \\
5 & 0.9391 & 0.9424 & 0.9375 & $0.17 \%$ \\
6 & $*$ & 1.194 & 1.189 & $\mathrm{n} / \mathrm{a}$ \\
7 & $*$ & 1.245 & 1.245 & $\mathrm{n} / \mathrm{a}$ \\
8 & 1.289 & 1.284 & 1.282 & $0.54 \%$ \\
9 & 1.428 & 1.455 & 1.448 & $1.40 \%$ \\
10 & 1.458 & 1.633 & 1.626 & $11.52 \%$ \\
\hline *Failed to identify as no dominant peaks observed &
\end{tabular}


Again, obvious difference existed beyond $1.5 \mathrm{~Hz}$. Further, Table 2 shows the identified first 10 natural frequencies using the worst segment, the best segment, and the whole data session. In the 6th and 7th modes, noisy peaks are found for the dataset with weak randomness and no resonance could be determined. Worse, the frequency of the 10th mode is shifted by $11.52 \%$ comparing to the one estimated from the optimal dataset.

\section{Conclusion}

The assumption of Gaussian white noise for an OMA method is important and has yet been emphasised in literature. By analysing the raw modal test data of two case-study structures, this paper has addressed the impact of the randomness of the measurements in OMA-based identification methods, and has proposed a technique to filter the optimal data segment. One the one hand, the modal identification can be misled particularly at high frequencies if the data have high Kurtosis values. On the other hand, the proposed randomness check with the windowing strategy is able to filter out the data segment which is most likely of a Gaussian white noise distribution. The proposed method is straightforward and can be applied as a processing step prior to modal identification. The influence and the technique of data selection on other modal identification methods will be focused in future work.

\section{Acknowledgement}

The presented work is financially aided by the Special Supportive Grant for Returned Overseas Scholars (No. OTP-2014-003) from Beijing Academy of Science and Technology.

\section{References}

1. T. Chan, D. P. Thambiratnam, Structural Health Monitoring in Australia (2011)

2. A. A. Shama, J. B. Mander, S. S. Chen, A. J. Aref, Ambient vibration and seismic evaluation of a cantilever truss bridge. Eng. Struct. 23, 10 1281$1292(2001)$

3. J. W. Brownjohn, F. Magalhaes, E. Caetano, A. Cunha, Ambient vibration re-testing and operational modal analysis of the Humber Bridge. Eng. Struct. 32, 8, 2003-2018 (2010)

4. C. Z. Chrysostomou, T. Dernetriou, A. Stassis, Health-monitoring and system-identification of an ancient aqueduct. Smart Struct. \&Sys. 4, 2 183-194 (2008)

5. S. El-Borgi, S. Choura, M. Neifar, H. Smaoui, M. S. Majdoub, D. Cherif, Seismic vulnerability assessment of a historical building in Tunisia. Smart Struct. \& Sys. 4, 2 209-220 (2008)

6. S. El-Borgi, M. Neifar, M. B. Jabeur, D. Cherif, H. Smaoui, Use of copper shape memory alloys in retrofitting historical monuments. Smart Struct. \& Sys. 4, 2 247-259 (2008)

7. A. El-Attar, A. Saleh, I. El-Habbal, A. H. Zaghw, A. Osman, The use of SMA wire dampers to enhance the seismic performance of two historical Islamic minarets, Smart Struct. \&Sys. 4, 2 221-232 (2008)

8. F. Magalhães, Á. Cunha, Explaining operational modal analysis with data from an arch bridge. Mech. Sys. \& Sig. Proc. 25, 5, 1431-1450 2011

9. R. Brincker, L. Zhang, L., P. Andersen, Modal identification from ambient responses using frequency domain decomposition, Proc. 18th int'l modal anal. Conf., 1, 625-630 San Antonio, TX. (2000)

10. H. Herlufsen, S. Gade, N. Møller, P. Andersen, Identification techniques for operational modal analysis - An overview and practical experiences. IMAC-XXIV: Conf. \& Expo. on Struct. Dyna. St Louis, MO: Curran Associates, Inc. (2006)

11. P. D. Welch, The use of the fast fourier transform for the estimation of power spectra. IEEE Trans. 15, 7073 (1967)

12. D. N. Joanes, C. A. Gill, Comparing measures of sample skewness and kurtosis. J. Royal Statistical Society Series D: The Statistician, 47, 1 183-189 (1998)

13. SVS (Structural Vibration Solutions A/S). How to set up the first measurement, help manual of ARTeMIS Extractor. Aalborg, East Denmark (2011).

14. L. Wang, Innovative Damage Assessment of Steel Truss Bridges using Modal Strain Energy Correlation, PhD Thesis, Queensland Univ. of Tech. (2012)

15. China Association for Engineering Construction Standardization, Design standard for structural health monitoring system (CECS 333: 2012), China Architecture \& Building Press, (2012)

16. H. Li, S. L. Li, J. P. Ou, H. W. Li, Modal identification of bridges under varying environmental conditions: Temperature and wind effects. Struct. Ctrl \& Health Monit. 17, 5, 495-512 (2010) 